\title{
Energy-Efficient Resource Allocation for Orthogonal Multi-antenna Multi-carrier Channel
}

\author{
Fabien Héliot, Muhammad Ali Imran, and Rahim Tafazolli \\ Centre for Communication Systems Research (CCSR), Faculty of Electronics \& Physical Sciences, \\ University of Surrey, Guildford GU2 7XH, UK. Email: F.Heliot@surrey.ac.uk
}

\begin{abstract}
Energy efficiency (EE) is growing in importance as a key performance indicator for designing the next generation of communication systems. Equally, resource allocation is an effective approach for improving the performance of communication systems. In this paper, we propose a low-complexity energyefficient resource allocation method for the orthogonal multiantenna multi-carrier channel. We derive explicit formulations of the optimal rate and energy-per-bit consumption for the per-antenna transmit power constrained and per-antenna rate constrained EE optimization problems as well as provide a lowcomplexity algorithm for optimally allocating resources over the orthogonal multi-antenna multi-carrier channel. We then compare our approach against a classic optimization tool in terms of energy efficiency as well as complexity, and results indicate the optimality and low-complexity of our approach. Comparing EE-optimal with spectral efficiency and power optimal allocation approaches over the orthogonal multi-antenna multi-carrier channel indicates that the former provides a good trade-off between power consumption and sum-rate performances.

Index Terms-Energy efficiency, resource allocation, MIMO, multi-carrier, realistic power model.
\end{abstract}

\section{INTRODUCTION}

Network operators not only require the next generation of communication systems to be more spectrally efficient, as with all the previous generations, but to also be more energyefficient for ensuring both the economical and environmental sustainability of their activity. Thus, energy efficiency (EE) is growing in importance as a key performance indicator such that it has lately attracted a lot of research interests [1]-[9].

Given that resource allocation and link adaptation are effective techniques for improving the performance of communication systems, these techniques have been thoroughly investigated in the past but mainly from a spectral efficiency (SE) or peak rate performance perspective. With the growing importance of $\mathrm{EE}$ in communication systems, resource allocation based on the EE criterion is becoming a popular research topic [3]-[9]. For instance, the work in [4] proposed an iterative gradient search algorithm for obtaining the EEoptimal resource allocation in the uplink of an orthogonal single-antenna multi-carrier/user channel. This work was then revisited in [6] by considering a more realistic power model for the user equipment (UE). Whereas, an EE-optimal resource allocation scheme for single-antenna orthogonal frequency multiplexing (OFDM) system was derived in [5]. More recently, we have proposed low-complexity energy-efficient resource allocation algorithms for the single-antenna broadcast channel, orthogonal single-antenna multi-carrier/user channel and single-antenna multi-user multi-cell system in [7], [8] and [9], respectively.

Contrary to the previous EE-based resource allocation works in [3]-[9], we here assume that the base station (BS) as well as $\mathrm{UE}(\mathrm{s})$ have multiple antennas and propose a low-complexity energy-efficient method for optimally allocating resources in the orthogonal multi-input multi-output (MIMO) multicarrier/user channel. More specifically, we extend our work of [8] for the MIMO scenario by considering a realistic multiantenna power consumption model and derive explicit formulations of the optimal rate and energy-per-bit consumption for the per-antenna transmit power constrained and per-antenna rate constrained EE optimization problems. In Section II, we first recall the per-subchannel rate and power formulations over the orthogonal MIMO multi-carrier/user channel, detail the power consumption models for both the BS and UE(s), and formulate the energy-per-bit consumption. In Section III, we first introduce the two optimization problems that are solved in this paper, i.e. minimizing the energy consumption over the orthogonal MIMO multi-carrier/user channel when considering a per-antenna power or rate constraint, and solve them by means of closed-form expressions. Based on these expressions, we provide a low-complexity algorithm for optimally allocating the power and rate in an energy efficient manner. In Section IV, we numerically show the optimality and low-complexity of our method in comparison with a traditional convex optimization method. As an application, we compare the EE-optimal, SEoptimal and power-optimal allocation methods and our results indicate that the former provides a good trade-off between power consumption and sum-rate performances. Conclusions are drawn in Section V.

\section{System And Power Consumption Models}

\section{A. System Model}

We consider the orthogonal MIMO multi-carrier/user channel, where $N K$ independent subchannels are used for transmitting data between a BS and one or several users. Moreover, we assume that each orthogonal subchannel is affected by block fading and that perfect channel state information is available at both the transmitter and receiver ends such that the achievable rate of the $(n, k)$-th parallel subchannel can be expressed as

$$
\mathcal{C}_{n, k}=\log _{2}\left(1+\frac{g_{n, k} p_{n, k}}{\Gamma \sigma^{2}}\right)
$$

which corresponds to the $(n, k)$-th rate of a MIMO-OFDM system with $N$ spatial and $K$ frequency flat subchannels [10] 
as well as of a $K$-user $N \times N$ MIMO-OFDMA transmission over a frequency-selective and block faded channel. In equation (1), $g_{n, k}$ is the channel gain over the $(n, k)$-th subchannel, $\sigma^{2}$ is the noise power, and $\Gamma$ represents the signal-to-noise ratio gap between the theoretical achievable rate and a practical coding and modulation scheme as in [4]. Conversely, the transmit power related to the $(n, k)$-th subchannel, $p_{n, k}$, can be expressed as

$$
p_{n, k}=\Gamma \sigma^{2}\left(2^{\mathcal{C}_{n, k}}-1\right) g_{n, k}^{-1},
$$

according to (1), such that the total transmit power can then be given by

$$
P(\mathcal{C})=\sum_{n=1}^{N} \sum_{k=1}^{K} a_{n, k}^{-1}\left(2^{\mathcal{C}_{n, k}}-1\right),
$$

where $\mathcal{C}=\left[\mathcal{C}_{1,1}, \ldots, \mathcal{C}_{1, K}, \mathcal{C}_{2,1}, \ldots, \mathcal{C}_{N, K}\right] \succeq 0$ and $a_{n, k} \triangleq$ $\left(\Gamma \sigma^{2}\right)^{-1} g_{n, k}$.

\section{B. Power consumption model and EE-SE trade-off formulation}

Even though BSs and UEs are different in their architectures and components, it has been shown in [2] and [4], respectively, that their power consumptions can be modelled as $P_{\text {in }}=\Delta P+$ $P^{C i}$, i.e. a linear relation between the consumed and transmit powers. Given that each antenna has its own RF chain [2], this model has been refined for the MIMO setting in [11] as

$$
P_{\text {in }}=t\left(\Delta P+P^{C i p A}\right)+P^{C i},
$$

where $\Delta$ is the radio frequency (RF) dependent slope of the power model, $P^{C i p A}$ is the per antenna circuit power, $t$ is the number of transmit antennas and $P^{C i}$ is the circuit power that is not dependent on $t$. In addition, the transmit power, $P$, is such that $P \in\left[0, P_{n}^{\max }\right]$ with $P_{n}^{\max }$ being the per-antenna maximum transmit power. Consequently, the total consumed power in the downlink or uplink of a multi-antenna multicarrier/user system can be linearly abstracted as

$$
P_{\Sigma}(\mathcal{C})=P_{\mathrm{c}}+\Delta P(\mathcal{C})
$$

when assuming the power model in (4) for the BS and UE(s), and where $P_{\mathrm{c}}=t P_{\mathrm{BS}}^{C i p A}+P_{\mathrm{BS}}^{C i}+\varsigma \kappa\left(r P_{\mathrm{UE}}^{C i p A}+P_{\mathrm{UE}}^{C i}\right)$ or $P_{\mathrm{c}}=\kappa\left(t P_{\mathrm{UE}}^{C i p A}+P_{\mathrm{UE}}^{C i}\right)+\varsigma\left(r P_{\mathrm{BS}}^{C i p A}+P_{\mathrm{BS}}^{C i}\right)$ in the downlink or uplink scenario, respectively. Moreover, $r$ is the number of receive antennas, $\kappa=1$ and $K$ in the OFDM and OFDMA settings, respectively; whereas $\varsigma$ characterizes the ratio between transmission and reception overhead powers with $0 \leq \varsigma \leq 1$. Intuitively, less overhead power is necessary for receiving than for transmitting signals. Note that $N=\min \{t, r\}$.

According to [12], the energy consumption, $E_{b}$, or EE, $1 / E_{b}$, can simply be defined as a ratio between the total consumed power and the sum-rate such that the EE-SE tradeoff of MIMO multi-carrier/user systems with full channel state information can be expressed according to (1), (3) and (5) as

$$
E_{b}(\mathcal{C}) \triangleq \frac{P_{\Sigma}(\mathcal{C})}{W \sum_{n=1}^{N} \sum_{k=1}^{K} \mathcal{C}_{n, k}}=\frac{P_{c}+\Delta \sum_{n=1}^{N} P_{n}(\mathcal{C})}{\sum_{n=1}^{N} R_{n}(\mathcal{C})}
$$

where $P_{n}(\mathcal{C})=\sum_{k=1}^{K} a_{n, k}^{-1}\left(2^{\mathcal{C}_{n, k}}-1\right), \quad R_{n}(\mathcal{C})=$ $W \sum_{k=1}^{K} \mathcal{C}_{n, k}$, and $W$ is the channel bandwidth.

\section{EE OPTIMIZATION OVER THE ORTHOGONAL MIMO} MULTI-CARRIER CHANNEL

We have recently solved in [8] the following EE-based problems over the orthogonal single-antenna multi-carrier channel:

$$
\begin{aligned}
& \min _{\mathcal{C}} E_{b}(\mathcal{C}), \text { s.t. } \mathcal{C} \succeq 0 \text { and } \\
& \min _{\mathcal{C}} E_{b}(\mathcal{C}), \text { s.t. } \mathcal{C} \succeq 0, \sum_{n=1}^{N} P_{n}(\mathcal{C}) \leq P^{\max } .
\end{aligned}
$$

However, given that in a realistic BS each antenna has its own RF chain and, hence, power constraint, per-antenna power constraint is more relevant than sum-power constraint from an EE point of view. Thus, we here extend our work of [8] and aim at minimizing the energy consumption when considering a per-antenna power or rate constraint by solving

$$
\begin{aligned}
& \min _{\mathcal{C}} E_{b}(\mathcal{C}), \text { s.t. } \mathcal{C} \succeq 0, P_{n}(\mathcal{C}) \leq P_{n}^{\max } \text { or } \\
& \min _{\mathcal{C}} E_{b}(\mathcal{C}), \text { s.t. } \mathcal{C} \succeq 0, R_{n}(\mathcal{C}) \geq R_{n}^{\min },
\end{aligned}
$$

respectively, for any $n \in \mathcal{N}=\{1, \ldots, N\}$.

We have proved in [8] that $E_{b}(\mathcal{C})$ is a convex function when $P_{c} \geq \Delta \sum_{m \in \mathcal{M}} \bullet a_{m}^{-1}$ and $\left|\mathcal{M}^{\bullet}\right| \geq 1$, where $m=$ $(n-1) K+k, n \in \mathcal{N}, \mathcal{M}^{\bullet}=\left\{m \in\{1, \ldots, N K\} \mid \mathcal{C}_{m}^{\bullet}>0\right\}$ is the optimal set of allocated subchannel indices and $\mathcal{C}_{m}^{\bullet}$ is the optimal value of $\mathcal{C}_{m}$ in the unconstrained problem of (7a). As long as each antenna does not transmit at full power $P_{n}^{\max }$ or achieve its minimum rate requirement $R_{n}^{\min }$, the problems in (8) revert to the unconstrained problem in (7a). Given that $E_{b}$ in (6) is convex, it has a unique global minimum $E_{b}^{\bullet}$ which is obtained for $\mathcal{C}=\mathcal{C}^{\bullet}$, where $\mathcal{C}^{\bullet}$ is the EE-optimal unconstrained achievable rate vector.

\section{A. Power constrained EE optimization}

Let us define $\overline{\mathcal{N}}=\left\{n \in \mathcal{N} \mid P_{n}\left(\mathcal{C}^{\bullet}\right) \geq P_{n}^{\max }\right\}$ and $\underline{\mathcal{N}}=$ $\left\{n \in \mathcal{N} \mid P_{n}\left(\mathcal{C}^{\bullet}\right)<P_{n}^{\max }\right\}$ are the index sets of antenna which are power constrained and power unconstrained, respectively, when $\mathcal{C}=\mathcal{C}^{\bullet}$.

Proposition 1: In the case that at least one antenna transmits at full power when $\mathcal{C}=\mathcal{C}^{\bullet}$, the EE-optimal achievable rate of the $k$-th subchannel served by any power constrained antenna $n$ (i.e. $n \in \overline{\mathcal{N}}$ ) can be given in closed-form by

$$
\mathcal{C}_{n, k}^{\star}=\log _{2}\left(\frac{P_{n}^{\max }+\sum_{i \in \mathcal{K}_{n}^{\star}} a_{n, i}^{-1}}{K_{n}^{\star} a_{n, k}^{-1}}\right),
$$

for $k \in \mathcal{K}_{n}^{\star}$, where $\mathcal{K}_{n}^{\star}=\left\{k \in \mathcal{K}=\{1, \ldots, K\} \mid \mathcal{C}_{n, k}^{\star}>0\right\}$ is the optimal set of allocated subchannel index for antenna $n$ in the power constrained case, and $K_{n}^{\star}=\left|\mathcal{K}_{n}^{\star}\right|$ is the number of elements in $\mathcal{K}_{n}^{\star}$. Note that $\mathcal{C}_{n, k}^{\star}=0$ for $k \in \mathcal{K} \backslash \mathcal{K}_{n}^{\star}$. See proof in Section A of the Appendix.

Remark 1: Equation (9) indicates that the rate allocation of each power constrained antenna can be performed independently by means of a simple binary search on $K_{n}^{\star}$. In addition, it ensures that any power constrained antenna $n$ (i.e. $n \in \overline{\mathcal{N}}$ ) transmits over at least one subchannel, the one with the best channel gain, such that $1 \leq K_{n}^{\star} \leq K$. 


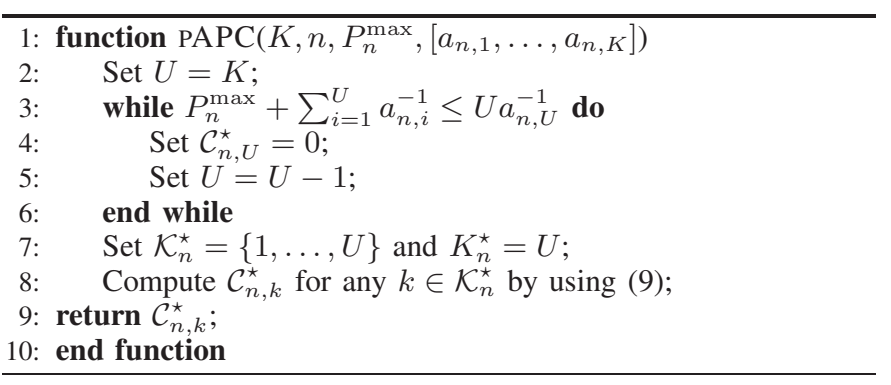

Proposition 2: In the case that at least one antenna transmits at full power when $\mathcal{C}=\mathcal{C}^{\bullet}$, the EE-optimal achievable rate of the $k$-th subchannel served by any unconstrained antenna $n$ (i.e. $n \in \underline{\mathcal{N}}$ ) can be expressed in closed-form as

$$
\mathcal{C}_{n, k}^{\star}=\log _{2}\left(a_{n, k} \frac{P_{c}^{\star}}{K^{\star}}\left[W_{0}\left(\frac{P_{c}^{\star}}{K^{\star}} e^{\frac{\ln (2) R_{c}^{\star}}{K^{\star}}-1}\right)\right]^{-1}\right),
$$

for $k \in \mathcal{K}_{n}^{\star}$, where $W_{0}$ denotes the real branch of the Lambert function [13], $K^{\star}=\sum_{i \in \underline{\mathcal{N}}} K_{i}^{\star}$,

$$
\begin{aligned}
& P_{c}^{\star}=\frac{P_{c}}{\Delta}+\sum_{i \in \overline{\mathcal{N}}} P_{i}^{\max }-\sum_{i \in \underline{\mathcal{N}}} \sum_{j=1}^{K_{i}^{\star}} a_{i, j}^{-1} \text { and } \\
& R_{c}^{\star}=\sum_{i \in \overline{\mathcal{N}}} \sum_{j=1}^{K} \mathcal{C}_{i, j}^{\star}+\sum_{i \in \underline{\mathcal{N}}} \sum_{j=1}^{K_{i}^{\star}} \log _{2}\left(a_{i, j}\right) .
\end{aligned}
$$

Note that $\mathcal{C}_{n, k}^{\star}=0$ for $k \in \mathcal{K} \backslash \mathcal{K}_{n}^{\star}$. See proof in Section B of the Appendix.

Remark 2: It can be remarked in (10) and (11) that the unconstrained part of the optimization is dependent on the result of the constrained part, such that the per-antenna power constrained EE optimization must be performed in two stages.

Based on equation (9), we propose the function "pAPC" for optimally allocating the power and rate in an energy efficient and low-complexity manner over the MIMO multi-carrier/user channel in the per-antenna power constrained scenario.

\section{B. Rate constrained EE optimization}

Similarly to the power constrained scenario, let use define $\overline{\mathcal{N}}=\left\{n \in \mathcal{N} \mid R_{n}\left(\mathcal{C}^{\bullet}\right)<R_{n}^{\min }\right\}$ and $\underline{\mathcal{N}}=\left\{n \in \mathcal{N} \mid R_{n}\left(\mathcal{C}^{\bullet}\right) \geq\right.$ $\left.R_{n}^{\min }\right\}$ are the index sets of antenna which are rate constrained and rate unconstrained, respectively, when $\mathcal{C}=\mathcal{C}^{\bullet}$.

Proposition 3: In the case that at least one antenna transmits below its target rate when $\mathcal{C}=\mathcal{C}^{\bullet}$, the EE-optimal achievable rate of the $k$-th user served by any rate constrained antenna $n$ (i.e. $n \in \overline{\mathcal{N}}$ ) can be given in closed-form by

$$
\mathcal{C}_{n, k}^{\star}=\log _{2}\left(a_{n, k}\right)+\frac{1}{K_{n}^{\star}}\left(\frac{R_{n}^{\min }}{W}-\sum_{i \in \mathcal{K}_{n}^{\star}} \log _{2}\left(a_{n, i}\right)\right),
$$

for $k \in \mathcal{K}_{n}^{\star}$, where $\mathcal{K}_{n}^{\star}$ is the optimal set of allocated user index for antenna $n$ in the rate constrained case. Note that $\mathcal{C}_{n, k}^{\star}=0$ for $k \in \mathcal{K} \backslash \mathcal{K}_{n}^{\star}$. Moreover, the EE-optimal achievable rate

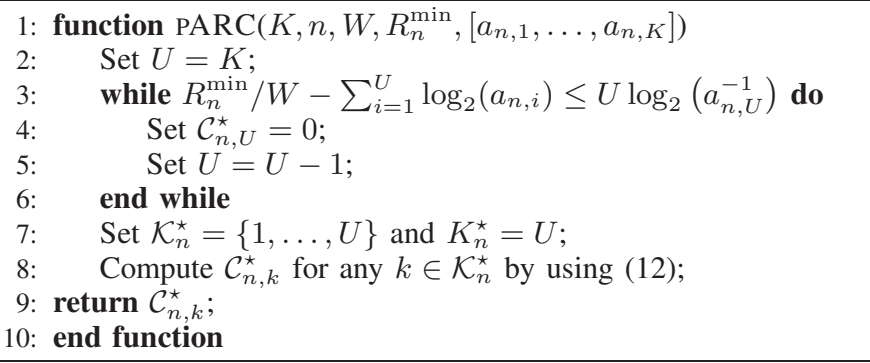

1: function $\operatorname{UNC}\left(K, \boldsymbol{\omega}, \widehat{\mathbf{a}}, P_{c}^{\star}, R_{c}^{\star}\right)$

Sort $\widehat{a}$ in descending order and obtain the index set $\pi$;

3: $\quad$ Set $U=\operatorname{length}(\widehat{\mathbf{a}}), \eta=1$;

4: while $\left\{\widehat{a}_{\pi_{U}} e^{\left(1+\frac{P_{c}^{\star} \widehat{a}_{U}}{U}\right)}<2^{\frac{R_{c}^{\star}}{U}}\right\} \&\{\eta==1\}$ do

5: $\quad$ Set $P_{c}^{\star}=P_{c}^{\star}+\widehat{a}_{\pi_{U}}^{-1}$ and $R_{c}^{\star}=R_{c}^{\star}+\log _{2}\left(\widehat{a}_{\pi_{U}}^{-1}\right)$;

6: $\quad$ Set $\mathcal{C}_{\omega_{j}, k}^{\star}=0$ for $j=\left\lfloor\left(\pi_{U}-1\right) / K\right\rfloor+1$ and $k=$

$\left(\pi_{U}-1\right) \bmod \{K\}+1$;

7: $\quad$ Set $U=U-1$;

8: $\quad$ if $U=0$ then

9: $\quad$ Set $\eta=0$ and $U=1$;

10: $\quad$ end if

11: end while

12: $\quad$ if $\eta==1$ then

13: $\quad$ Compute $\mathcal{C}_{\omega_{j}, k}^{\star}$ for $j=\left\lfloor\left(\pi_{i}-1\right) / K\right\rfloor+1, k=\left(\pi_{i}-1\right)$

$\bmod \{K\}+1$ and any $i \in\{1, \ldots, U\}$ by using (10) with $K^{\star}=U$;

14: end if

15: return $\mathcal{C}_{\omega_{j}, k}^{\star}$;

16: end function

of the $k$-th user served by any unconstrained antenna $n$ (i.e. $n \in \underline{\mathcal{N}}$ ) can be expressed in closed-form as in (10), but where

$$
\begin{aligned}
& P_{c}^{\star}=\frac{P_{c}}{\Delta}+\sum_{i \in \overline{\mathcal{N}}} \sum_{j=1}^{K} a_{i, j}^{-1}\left(2^{\mathcal{C}_{i, j}^{\star}}-1\right)-\sum_{i \in \underline{\mathcal{N}}} \sum_{j=1}^{K_{i}^{\star}} a_{i, j}^{-1} \text { and (13a) } \\
& R_{c}^{\star}=\sum_{i \in \overline{\mathcal{N}}} \frac{R_{i}^{\min }}{W}+\sum_{i \in \underline{\mathcal{N}}} \sum_{j=1}^{K_{i}^{\star}} \log _{2}\left(a_{i, j}\right) .
\end{aligned}
$$

Based on equation (12), we propose the function "pARC" for optimally allocating the power and rate in an energy efficient and low-complexity manner over the MIMO multi-carrier/user channel in the per-antenna rate constrained scenario.

\section{EE optimization algorithm}

Since $E_{b}$ in (6) is convex, it has a unique global minimum, which is obviously the best operation point in terms of EE. Consequently, our algorithm must first search for this optimal unconstrained energy-efficient point, i.e. $\mathcal{C}=\mathcal{C}^{\bullet}$ by using the "Unc" function, which is defined above. However, if this $\mathcal{C}^{\bullet}$ fails to meet the per-antenna rate or power constraint, then the allocation must be refined by using the "pARC" or "pAPC" functions, respectively, such that the optimal constrained energy-efficient point becomes $\mathcal{C}^{\star}$. The results of the "pARC" and "pAPC" functions must then be plugged back into the "Unc" function for further refining the optimization until no more antennas are constrained or all the antennas are constrained, as it is indicated in Algorithm 1. 


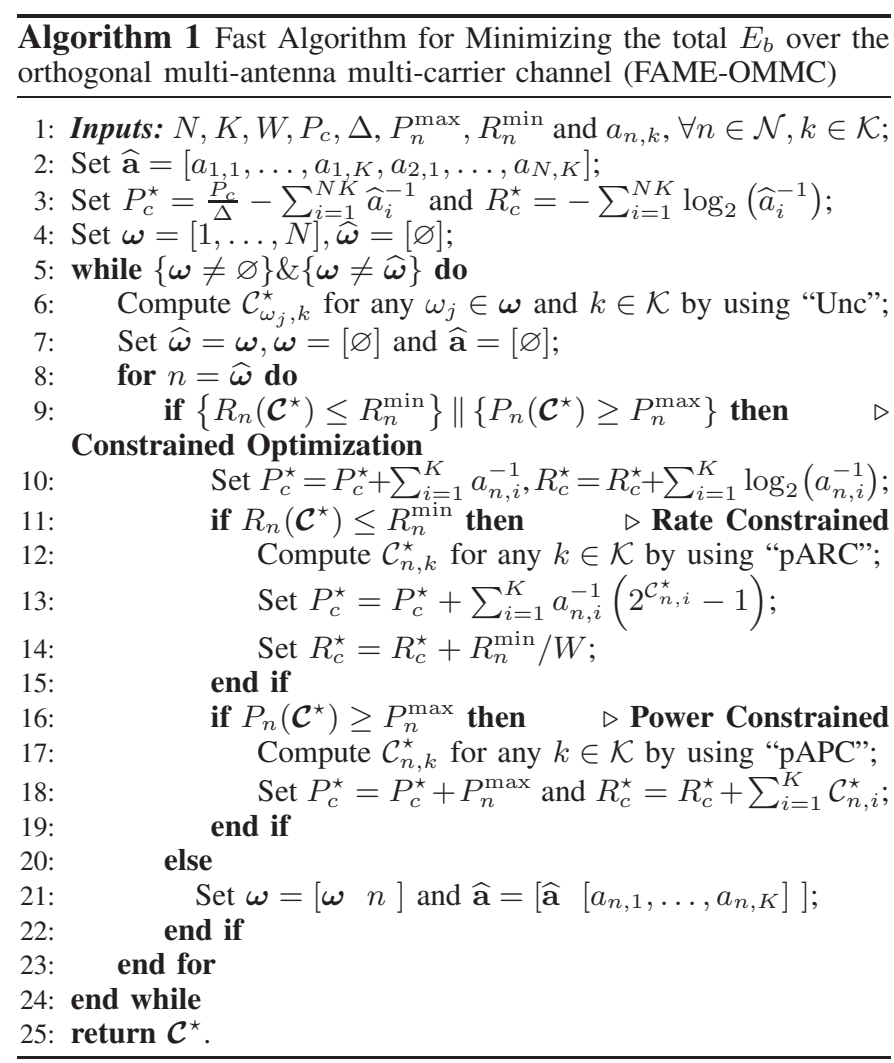

Our low-complexity algorithm in Algorithm 1 clearly reflects this two-stage procedure, where the unconstrained search is performed at line 6 and the rate and/or power constrained updates are performed at lines 12 and 18, respectively. As in the water-filling algorithm, our algorithm in Algorithm 1 is based on closed-form expressions and unidimensional searches, which makes its computational complexity low. However, it has an iterative structure (while loop in line 5) for ensuring optimality; it requires on average 2.5 iterations to converge. We assume here that the channel gains $a_{n, k}$ are sorted in descending order for each antenna prior to using our algorithm, such that $a_{n, 1} \geq a_{n, 2} \geq \ldots \geq a_{n, K}$ for any $n \in \mathcal{N}$. In addition, $\lfloor$.$\rfloor and mod stand for the "floor" and modulo$ operators in lines 6 and 13 of the "Unc" function.

\section{Numerical Results And Discussions}

In order to demonstrate the reliability of our algorithm for optimally allocating power and rate over the orthogonal MIMO multi-carrier/user channel in an energy-efficient manner, we compare in Figs. 1 and 2 the results, averaged over 1000 runs, of our FAME-OMMC algorithm against the Matlab "fmincon" function in terms of energy-per-bit performance (upper graph) as well as relative computational complexity (lower graph). We define the relative computational complexity between these two methods as the ratio of the "fmincon" execution time to our algorithm execution time. Given that (6) is a convex function, the minimization problems in (8a) and (8b) can be optimally solved via traditional convex optimization tools such as the Matlab "fmincon" function and, hence, we use the
TABLE I: Power parameter values

\begin{tabular}{|c||c|c|c|c|}
\hline Parameters & $\Delta$ & $P^{C i p A}(\mathrm{~W})$ & $P^{C i}(\mathrm{~W})$ & $P_{n}^{\max }(\mathrm{W})$ \\
\hline BS & $4.7[2]$ & 100 & 180 & $20[2]$ \\
\hline UE & - & 0.03 & 0.07 & - \\
\hline
\end{tabular}

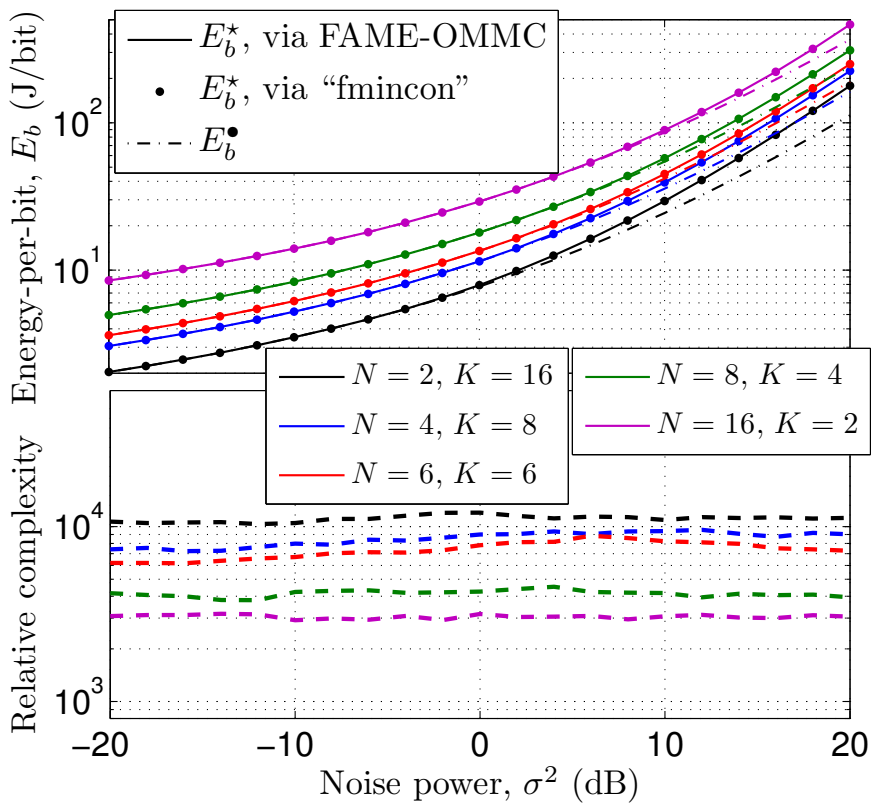

Fig. 1: Comparison of our FAME-OMMC algorithm with "fmincon" in terms of $E_{b}$ and complexity for various values of $N$ and $K$ with $P_{n}^{\max }=20 \mathrm{~W}, R_{n}^{\min }=0 \mathrm{bit} / \mathrm{s}, \forall n \in \mathcal{N}$.

latter as a benchmark. Figure 1 depicts the EE-optimal perantenna power constrained results of both our Algorithm and "fmincon" as a function of noise power for $P_{n}^{\max }=20 \mathrm{~W}$ and $R_{n}^{\min }=0 \mathrm{bit} / \mathrm{s}, \forall n \in \mathcal{N}$, as well as various values of $N$ and $K$ when considering the power parameter values of Table I, $W=1$ and $\varsigma=1 / 2$. Whereas, Fig. 2 shows the EE-optimal per-antenna rate constrained results of both our Algorithm and "fmincon" for $P_{n}^{\max }=10^{6} \mathrm{~W}, R_{n}^{\min }=20 \mathrm{bit} / \mathrm{s}, \forall n \in \mathcal{N}$, and the same other parameters as in Fig. 1.

Both Figs. 1 and 2 clearly indicate the tight match between our FAME-OMMC algorithm results and the "fmincon" function, which, in turn, graphically confirms the great accuracy and reliability of our energy-efficient resource allocation algorithm. Comparing the EE-optimal unconstrained results $E_{b}^{\bullet}$, i.e. for $P_{n}^{\max }=10^{6} \mathrm{~W}$ and $R_{n}^{\min }=0 \mathrm{bit} / \mathrm{s}, \forall n \in$ $\mathcal{N}$, with the constrained results indicates that the EE-based resource allocation becomes constrained when the channel quality degrades, i.e. when the noise power increases; indeed, we know from [14] that over the MIMO channel the EEoptimal power increases with the noise power. Whereas, the relative computational complexity results in the lower part of Figs. 1 and 2 graphically show the low-complexity of our algorithm in comparison with a conventional gradient-search algorithm; indeed, our algorithm can reduce the computational complexity by at least three orders of magnitude, i.e. $>2000$ times, in every presented settings. 


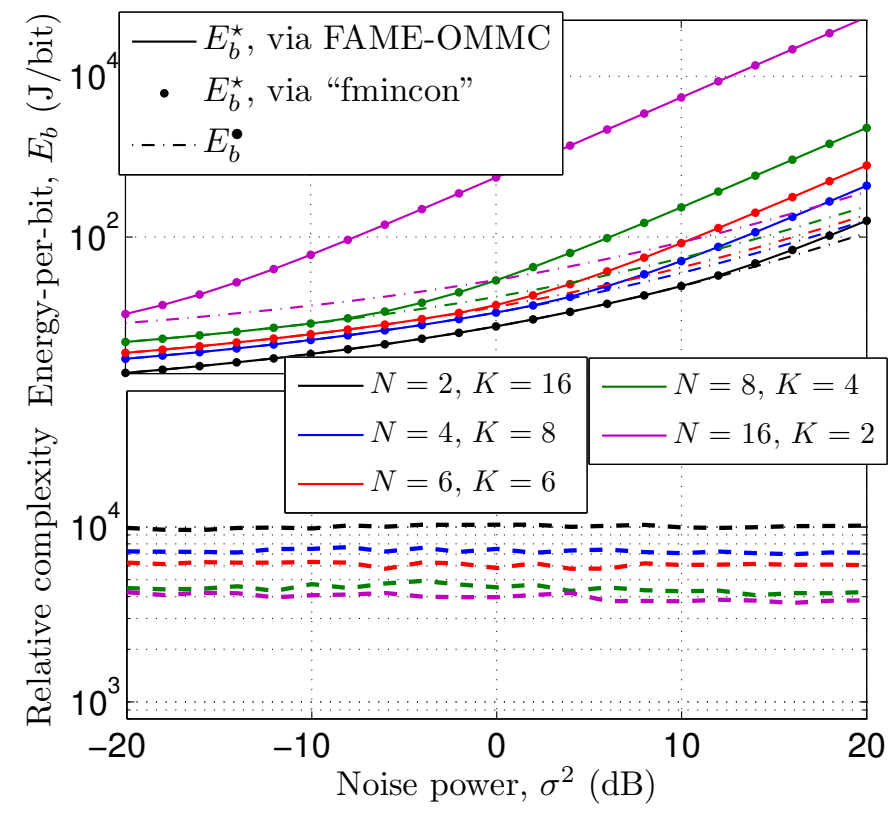

Fig. 2: Comparison of our FAME-OMMC algorithm with "fmincon" in terms of $E_{b}$ and complexity for various values of $N$ and $K$ with $P_{n}^{\max }=10^{6} \mathrm{~W}, R_{n}^{\min }=20 \mathrm{bit} / \mathrm{s}, \forall n \in \mathcal{N}$.

TABLE II: System parameter values

\begin{tabular}{|c||c|c|}
\hline & $f_{c}$ & $2.1 \mathrm{GHz}$ \\
& $W$ & $10 \mathrm{MHz}$ \\
& $N_{0}$ & $-165.2 \mathrm{dBm} / \mathrm{Hz}$ \\
System & $G_{T x R x}$ & $14 \mathrm{dBi}$ \\
parameters & $h_{\mathrm{BS}}$ & 25 or $35 \mathrm{~m}$ if ISD $\leq$ or $>d_{\mathrm{BP}}$ \\
{$[15]$} & $h_{\mathrm{av}}$ & 20 or $5 \mathrm{~m}$ if $\mathrm{ISD} \leq$ or $>d_{\mathrm{BP}}$ \\
& $W_{\mathrm{St}}$ & $20 \mathrm{~m}$ \\
& $h_{\mathrm{UT}}$ & $1.5 \mathrm{~m}$ \\
\hline
\end{tabular}

As an application, we study the trade-off between power, energy and rate in Fig. 3 by comparing our FAME-OMMC algorithm with rate adaptation, i.e. SE-optimal resource allocation subject to a per-antenna power constraint, and margin adaptation, i.e. power-optimal resource allocation subject to a per-antenna rate constraint, when considering a realistic downlink setting with both path-loss and small scale fading. We utilize the following pathloss model such that the pathloss between the $\mathrm{BS}$ and the $k$-th user is given by

$$
\rho_{k}=10^{\left(G_{\mathrm{TxRx}}-\mathrm{PL}\left(d_{k}\right)\right) / 10},
$$

where $G_{\mathrm{TxRx}}$ is the antenna gain of the transmission and $\operatorname{PL}(d)=\operatorname{Pb}_{\mathrm{LOS}}(d) \mathrm{PL}_{\mathrm{LOS}}(d)+\left(1-\mathrm{Pb}_{\mathrm{LOS}}\right) \mathrm{PL}_{\mathrm{NLOS}}(d)$ is the distance dependent path-loss function. In addition, $\mathrm{Pb}_{\mathrm{LOS}}$ is the line-of-sight (LOS) probability, $\mathrm{PL}_{\mathrm{LOS}}(d)$ and $\mathrm{PL}_{\mathrm{NLOS}}(d)$ are the LOS and non-LOS (NLOS) path-loss functions, whose values can be found in Table 27 of [15]. Note that we considered here the urban macro (UMa) setting in Table 27 of [15] for the parameter values given in Table II, where $d_{\mathrm{BP}}$ is the breakpoint distance [15].

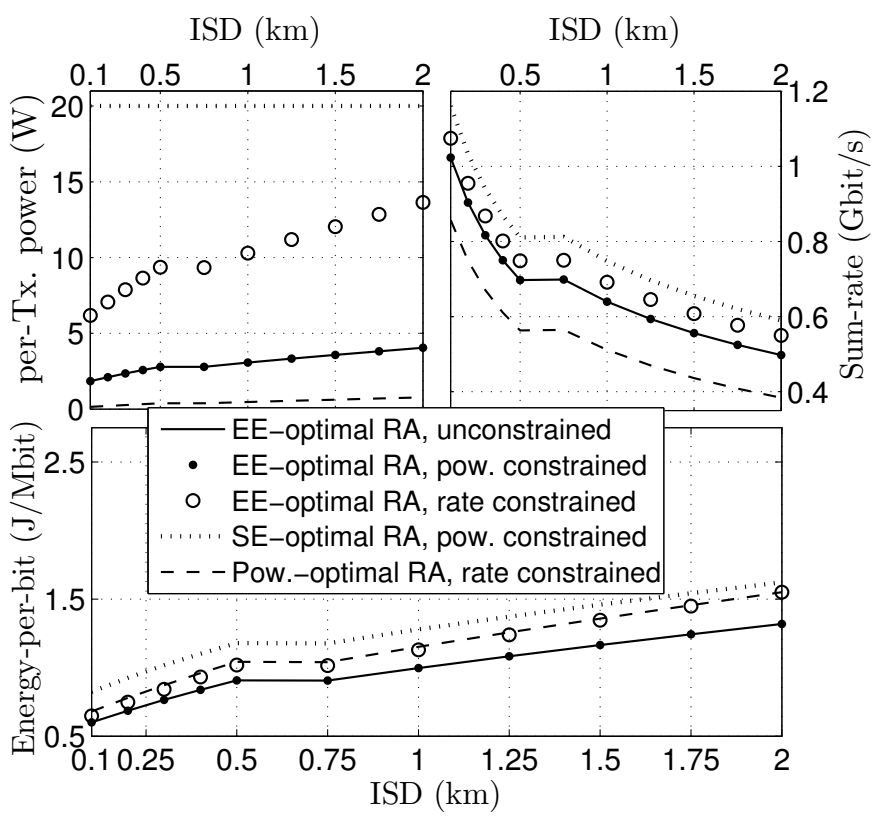

Fig. 3: Performance comparison of the EE-optimal, SEoptimal and power-optimal resource allocations as a function of the ISD for $N=4$ and $K=256$ users.

Relying on this pathloss model, the power parameter values of Table I, and the system parameter values of Table II with $\sigma^{2}=N_{0} W$, we plot the EE-optimal unconstrained, power and rate per-antenna constrained as well as SE-optimal perantenna power constrained and power-optimal per-antenna rate constrained resource allocation performances in terms of perantenna transmit power, sum-rate and energy consumption for $N=4$ and $K=256$ uniformly distributed users with one user per subcarrier. In the power-optimal rate constrained resource allocation, we set $R_{n}^{\min }=0.9 \min _{k}\left\{R_{k}\left(\mathcal{C}^{\bullet}\right)\right\}, \forall n \in \mathcal{N}$, and in EE-optimal rate constrained resource allocation, we set $R_{n}^{\min }=\max _{k}\left\{R_{k}\left(\mathcal{C}^{\bullet}\right)\right\}, \forall n \in \mathcal{N}$. The results indicate that our EE-optimal resource allocation provides the lowest energy-per-bit consumption in comparison with the power and SE-optimal allocations. Our method reduces the energy-perbit consumption by reducing the transmit power by more than $80 \%$ in comparison with the SE-optimal allocation. The power-optimal allocation provides an even larger reduction in transmit power but at the expense of a lower sum-rate, which results in a higher energy-per-bit consumption. Moreover, in this particular setting, the EE-optimal unconstrained and power constrained allocations are the same since none of the antennas have to transmit at full power for minimizing the energy consumption.

\section{CONCLUSion}

In this paper, a low-complexity energy-efficient resource allocation methods for the orthogonal MIMO multi-carrier/user channel has been proposed when considering a realistic multiantenna power model. We have derived explicit formulations of the optimal rate and energy-per-bit consumption for the 
per-antenna transmit power constrained and per-antenna rate constrained EE optimization problems. Based on these formulations, we have designed a low-complexity EE-optimal algorithm for allocating resources over the orthogonal MIMO multi-carrier/user channel. Simulations have demonstrated that our method is both accurate and low-complexity when compared to a traditional convex optimization method. Our results have also showed that EE-based allocation can significantly reduce the consumed power and provide a good trade-off between power consumption and sum-rate performance.

\section{APPENDIX}

\section{A. Proof for Proposition 1}

Proof: In the case that at least one antenna transmits at full power when $\mathcal{C}=\mathcal{C}^{\bullet}$, the Lagrangian associated to the problem in $(8 \mathrm{a})$ is equivalent to

$$
\begin{aligned}
\mathcal{L}(\mathcal{C}, \widehat{\boldsymbol{\mu}}) & =\left[P_{c}+\Delta\left(\sum_{n \in \overline{\mathcal{N}}} P_{n}^{\max }+\sum_{n \in \underline{\mathcal{N}}} P_{n}(\mathcal{C})\right)\right] \\
& \times\left(\sum_{n=1}^{N} R_{n}(\mathcal{C})\right)^{-1}+\sum_{n \in \overline{\mathcal{N}}} \widehat{\mu}_{n}\left(P_{n}(\mathcal{C})-P_{n}^{\max }\right),
\end{aligned}
$$

where $\widehat{\mu}_{n}$ is a slack variable and $\widehat{\boldsymbol{\mu}}=\left[\widehat{\mu}_{1}, \ldots, \widehat{\mu}_{N}\right]$. According to (15), solving $\nabla \mathcal{L}\left(\mathcal{C}^{\star}, \widehat{\boldsymbol{\mu}}^{\star}\right)=\mathbf{0}$ yields

$$
E_{b}\left(\mathcal{C}^{\star}\right)=\left\{\begin{array}{c}
\mu_{n}^{\star} \Delta \frac{\partial P_{n}\left(\mathcal{C}^{\star}\right)}{\partial \mathcal{C}_{n, k}}\left[\frac{\partial R_{n}\left(\mathcal{C}^{\star}\right)}{\partial \mathcal{C}_{n, k}}\right]^{-1}, \text { if } n \in \overline{\mathcal{N}} \text { or } \\
\Delta \frac{\partial P_{n}\left(\mathcal{C}^{\star}\right)}{\partial \mathcal{C}_{n, k}}\left[\frac{\partial R_{n}\left(\mathcal{C}^{\star}\right)}{\partial \mathcal{C}_{n, k}}\right]^{-1}, \text { if } n \in \underline{\mathcal{N}},
\end{array}\right.
$$

where $\mu_{n}^{\star}=\widehat{\mu}_{n}^{\star} \sum_{n=1}^{N} R_{n}\left(\mathcal{C}^{\star}\right)$ with $R_{n}\left(\mathcal{C}^{\star}\right)$ being a constant scalar value. Given that $\frac{\partial P_{n}\left(\mathcal{C}^{\star}\right)}{\partial \mathcal{C}_{n, k}}=\ln (2) a_{n, k}^{-1} 2^{\mathcal{C}_{n, k}^{\star}}$ and $\frac{\partial R_{n}\left(\mathcal{C}^{\star}\right)}{\partial \mathcal{C}_{n, k}}=W$, equation (16) implies that

$$
a_{n, i}^{-1} 2^{\mathcal{C}_{n, i}^{\star}}=a_{n, k}^{-1} 2^{\mathcal{C}_{n, k}^{\star}}
$$

for any $n \in \mathcal{N}$ and $i, k \in \mathcal{K}_{n}^{\star}$. Since $P_{n}\left(\mathcal{C}^{\star}\right)=P_{n}^{\max }$ for $n \in \overline{\mathcal{N}}$, it implies with $P_{n}(\mathcal{C})=\sum_{i=1}^{K} a_{n, i}^{-1}\left(2^{\mathcal{C}_{n, i}}-1\right)$ and (17) that

$$
P_{n}^{\max }=K_{n}^{\star} a_{n, k}^{-1} 2^{\mathcal{C}_{n, k}^{\star}}-\sum_{i \in \mathcal{K}_{n}^{\star}} a_{n, i}^{-1}
$$

for any $n \in \overline{\mathcal{N}}$ when $\mathcal{C}=\mathcal{C}^{\star}$. Equation (9) is then a simple reformulation of (18).

\section{B. Proof for Proposition 2}

Proof: Equation (16) also implies that

$$
a_{i, j}^{-1} 2^{\mathcal{C}_{i, j}^{\star}}=\mu_{n}^{\star} a_{n, k}^{-1} 2^{\mathcal{C}_{n, k}^{\star}}
$$

for any $i \in \underline{\mathcal{N}}, n \in \overline{\mathcal{N}}, j \in \mathcal{K}_{i}^{\star}$ and $k \in \mathcal{K}_{n}^{\star}$. By using (19), i.e. substituting $a_{i, j}^{-1} 2^{\mathcal{C}_{i, j}^{\star}}$ with $\mu_{n}^{\star} a_{n, k}^{-1} 2^{\mathcal{C}_{n, k}^{\star}}$, into (6), the latter can be reformulated as

$$
E_{b}\left(\mathcal{C}^{\star}\right)=\frac{\Delta\left[P_{c}^{\star}+\left(\sum_{i \in \underline{N}} K_{i}^{\star}\right) \mu_{n}^{\star} a_{n, k}^{-1} 2^{\mathcal{C}_{n, k}^{\star}}\right]}{W\left[R_{c}^{\star}+\left(\sum_{i \in \underline{N}} K_{i}^{\star}\right)\left(\mathcal{C}_{n, k}^{\star}+\log _{2}\left(\mu_{n}^{\star} a_{n, k}^{-1}\right)\right)\right]},
$$

where $P_{c}^{\star}$ and $R_{c}^{\star}$ are given in (11a) and (11b), respectively. Moreover we know from (16a) that

$$
E_{b}\left(\mathcal{C}^{\star}\right)=\ln (2) \Delta \mu_{n}^{\star} W^{-1} a_{n, k}^{-1} 2^{\mathcal{C}_{n, k}^{\star}} .
$$

By inserting (21) into (20), we obtain an equality solely in terms of the unknown variable $\mu_{n}^{\star}$ that yields after some simplifications

$$
\mu_{n}^{\star}=\frac{P_{c}^{\star}}{K^{\star} a_{n, k}^{-1} 2^{\mathcal{C}_{n, k}^{\star}}}\left[W_{0}\left(\frac{P_{c}^{\star}}{K^{\star}} e^{\frac{\ln (2) R_{c}^{\star}}{K^{\star}}-1}\right)\right]^{-1} .
$$

We finally obtain (10) by inserting (22) into (19).

\section{ACKNOWLEDGMENT}

The research leading to these results has received funding from the European Commission's Seventh Framework Programme FP7/2007-2013 under grant agreement n³18273project LEXNET.

\section{REFERENCES}

[1] H. Zhang et al., "Energy efficiency in communications," IEEE Commun. Mag., vol. 48, no. 11, pp. 48-79, Nov. 2010.

[2] G. Auer et al., "How Much Energy is Needed to Run a Wireless Network ?" IEEE Wireless Commun., vol. 18, no. 5, pp. 40-49, Oct. 2011.

[3] F. Meshkati, H. V. Poor, S. C. Schwartz, and N. B. Mandayam, "An Energy-Efficient Approach to Power Control and Receiver Design in Wireless Networks," IEEE Trans. Commun., vol. 5, no. 1, pp. 33063315, Nov. 2006.

[4] G. Miao, N. Himayat, and G. Y. Li, "Energy-Efficient Link Adaptation in Frequency-Selective Channels," IEEE Trans. Commun., vol. 58, no. 2, pp. 545-554, Feb. 2010.

[5] R. S. Prabhu and B. Daneshrad, "An Energy-efficient Water-filling Algorithm for OFDM Systems," in Proc. IEEE ICC'10, Dresden, Germany, Jun. 2010.

[6] C. Isheden and G. P. Fettweis, "Energy-Efficient Multi-Carrier Link Adaptation with Sum Rate-Dependent Circuit Power," in Proc. IEEE Globecom, Miami, USA, Dec. 2010.

[7] F. Héliot, M. A. Imran, and R. Tafazolli, "Energy-efficiency based resource allocation for the scalar broadcast channel," in Proc. IEEE WCNC, Paris, France, Apr. 2012.

[8] — "Energy-efficiency based resource allocation for the orthogonal multi-user channel," in Proc. IEEE VTC-Fall, Québec city, Canada, Sep. 2012.

[9] — , "Low-complexity energy-efficient coordinated resource allocation in cellular systems," IEEE Trans. Commun., vol. 61, no. 6, pp. 22712281, Jun. 2013.

[10] H. Bölcskei, D. Gesbert, and A. J. Paulraj, "On the Capacity of OFDMBased Spatial Multiplexing Systems," IEEE Trans. Commun., vol. 50, no. 2, pp. 225-234, Feb. 2002.

[11] F. Héliot, M. A. Imran, and R. Tafazolli, "On the energy efficiencyspectral efficiency trade-off over the MIMO Rayleigh fading channel," IEEE Trans. Commun., vol. 60, no. 5, pp. 1345-1356, May 2012.

[12] S. Verdu, "Spectral Efficiency in the Wideband Regime," IEEE Trans. Inf. Theory, vol. 48, no. 6, pp. 1319-1343, Jun. 2002.

[13] R. M. Corless, G. H. Gonnet, D. E. G. Hare, D. J. Jeffrey, and D. E. Knuth, "On the LambertW Function," Adv. Comput. Math., vol. 5, pp. 329-359, 1996.

[14] F. Héliot, M. A. Imran, and R. Tafazolli, "Energy-efficient power allocation for point-to-point MIMO systems over the Rayleigh fading channel," IEEE Wireless Commun. Lett., vol. 1, no. 4, pp. 304-307, Aug. 2012.

[15] A. Ambrosy et al., "D2.2: Definition and Parameterization of Reference Systems and Scenarios," INFSO-ICT-247733 EARTH (Energy Aware Radio and NeTwork TecHnologies), Tech. Rep., Jun. 2010. 